THE aim of this study was to determine the role of CC chemokine CCL6/C10 in acute inflammation. Intraperitoneal injection of thioglycollate increased peritoneal CCL6, which peaked at $4 \mathrm{~h}$ and remained elevated at $48 \mathrm{~h}$. Neutralization of CCL6 significantly inhibited the macrophage infiltration $(34-48 \%$ reduction), but not other cell types, without decreasing the other CC chemokines known to attract monocytes/macrophages. CCL6 was expressed in peripheral eosinophils and elicited macrophages, but not in elicited neutrophils. Peritoneal CCL6 level was not decreased in granulocyte-depleted mice where eosinophil influx was significantly impaired. Thus, CCL6 appears to contribute to the macrophage infiltration that is independent of other CC chemokines. Eosinophils pre-store CCL6, but do not release CCL6 in the peritoneum in this model of inflammation.

Key words: Acute inflammation, CC chemokines, Eosinophils, Monocytes/macrophages, Neutrophils

\section{Role of CC chemokine CCL6/C10 as a monocyte chemoattractant in a murine acute peritonitis}

\author{
Andrew M. LaFleur ${ }^{1,2}$, Nicholas W. Lukacs ${ }^{1}$, \\ Steven L. Kunkel ${ }^{1}$ and Akihiro Matsukawa ${ }^{1,3, C A}$ \\ ${ }^{1}$ Department of Pathology, University of Michigan \\ Medical School, Ann Arbor, Michigan, USA; \\ ${ }^{2}$ Michigan State University, Kalamazoo Center for \\ Medical Studies, Kalamazoo, Michigan, USA; and \\ ${ }^{3}$ Department of Pathology and Experimental \\ Medicine, Graduate School of Medical Sciences, \\ Kumamoto University, 1-1-1 Honjo, Kumamoto \\ 860-8556, Japan
}

${ }^{\mathrm{CA}}$ Corresponding Author
Tel: +81963735088
Fax: +81963735087
E-mail: matsu@kaiju.medic.kumamoto-u.ac.jp

\section{Introduction}

The inflammatory response is characterized by leukocyte infiltration into inflammatory foci, which is initiated and orchestrated by a variety of inflammatory mediators. Of these, chemokines are a group of cytokines that are chemotactic for specific types of leukocyte populations. ${ }^{1,2}$ Chemokines have been divided mainly into two subfamilies, CXC and CC chemokines, based on their sequence homology and the position of cysteine residues. ${ }^{3,4} \mathrm{CXC}$ chemokines are typically chemotactic for neutrophils, whereas CC chemokines attract and activate monocytes and lymphocytes. The role of CXC chemokine such as CXC8/IL-8 in a variety of inflammatory diseases has been extensively investigated. ${ }^{5}$ CC chemokines that include CCL2/MCP-1, CCL3/MIP- $1 \alpha$ and CCL22/MDC are detected in an early phase of acute inflammation, playing an essential role in the recruitment and activation of monocytes/macrophages. ${ }^{6-10}$

Murine CCL6/C10, a prototype CC chemokine, was initially identified as a transcript induced in bone marrow cells upon stimulation with granulocyte-macrophage colony-stimulating factor. ${ }^{11}$ It has shown that CCL6 is present in a variety of chronic inflammatory disorders including experimental demyelinating diseases, allergic airway inflammation, bleomycin-induced lung fibrosis and chronic peri- tonitis. ${ }^{12-15}$ These findings demonstrate an important role of CCL6 in chronic inflammation associated with macrophage infiltration. However, the precise role of CCL6 in acute inflammation remains to be elucidated.

The aim of the present study was to characterize the role of CCL6 in acute inflammation. For this purpose, we first examined the production kinetics of CCL6 in a murine model of acute peritonitis induced by thioglycollate injection. We then investigated whether CCL6 could contribute to the leukocyte infiltration in this model by neutralizing the endogenous CCL6 with antibodies against CCL6. Finally, we attempted to determine the major cellular sources of CCL6 using reverse transcription-polymerase chain reaction (RT-PCR), enzyme-linked immunosorbent assay (ELISA) and immunohistochemistry. The present data suggest that CCL6 is an important CC chemokine attracting monocytes/macrophages. Although CCL6 is pre-stored in circulating eosinophils, the CCL6 does not appear to be released in this particular model.

\section{Materials and methods}

Mice

Female CD-1 mice (6-8 weeks of age) were purchased from Jackson Laboratory (Bar Harbor, ME, USA) 
and housed in the animal care facility unit (University Laboratory of Animal Medicine). The animal use committee at the University of Michigan approved all studies.

\section{Peritonitis model}

The mice received intraperitoneal (i.p.) injection of 1 $\mathrm{ml}$ of a $2 \%$ thioglycollate broth (Difco Laboratories, Detroit, MI, USA). To neutralize CCL6, $0.5 \mathrm{ml}$ of antimurine CCL6 antiserum was i.p. injected $2 \mathrm{~h}$ prior to thioglycollate injection. Polyclonal anti-murine CCL6 antiserum was raised by immunizing rabbits with recombinant murine CCL6 (R\&D systems Inc., Minneapolis, MN, USA), as previously described. ${ }^{13,16}$ The antibodies had a neutralizing activity against murine recombinant CCL6 in vitro chemotaxis assay, and did not cross-react with a number of other murine cytokines and chemokines available (data not shown). As a control, pre-immune rabbit serum (0.5 $\mathrm{ml}$ ) was used. At appropriate time points following thioglycollate injection, mice were euthanized by cervical dislocation. Peritoneal lavages were collected by washing the peritoneum with $2 \mathrm{ml}$ of sterile saline, were centrifuged, and cell-free peritoneal fluids were stored at $-20^{\circ} \mathrm{C}$ for measurements of chemokines. Cell pellets were resuspended in saline, and the cell numbers were counted in a hemocytometer. Differential cell analysis was made after Diff-Quik staining of cytospin preparations.

\section{Granulocyte depletion}

Granulocytes were depleted using RB6-8C5 monoclonal antibody directed against Ly-6G, previously known as Gr-1, an antigen on the surface of murine granulocytes that includes neutrophils and eosinophils. ${ }^{17,18}$ A total of $100 \mu \mathrm{g}$ of RB6-8C5 was i.p. administered 1 day prior to thioglycollate injection. This resulted in peripheral blood granulopenia $(<50$ cells/ $\mu \mathrm{l}$ ) by days 1 and 3 after the administration, with a return of peripheral counts to pretreatment levels by day $5 .{ }^{19}$ Due to the granulopenia, the treatment effectively inhibited neutrophil and eosinophil infiltration in animal models of inflammation. ${ }^{19,20}$ Isotype-matched mouse IgG was used as a control.

\section{Isolation of granulocytes and macrophages}

Elicited granulocytes and macrophages were isolated from the peritoneal exudates after thioglycollate injection by Percoll gradient centrifugation. For this, exudates from five mice or two mice were combined in each experiment for granulocyte or macrophage isolation, respectively. Six independent experiments were performed. Peripheral granulocytes were isolated from heparinized untreated mice blood (10 mice each, three independent experiments) by dex- tran sedimentation, followed by Percoll gradient centrifugation and hypotonic lysis of erythrocytes. Diff-Quik staining of cytospin preparations showed that the purity of granulocytes and macrophages in each experiment was $>92 \%$ and $>94 \%$, respectively. Resident peritoneal macrophages were harvested from untreated mice, and the adherent cells were used. Isolated cells were used for RT-PCR and ELISA. For ELISA assay, cells were extracted with phosphatebuffered saline containing 1\% Triton X-100 and protease inhibitors (Roche Applied Science, Basel, Switzerland).

\section{RT-PCR}

Total RNA was isolated from cells using Trizol reagent (Life Technologines, Grand Island, NY, USA) according to the manufacturer's instructions. First-strand cDNA was synthesized from $1 \mu \mathrm{g}$ of total RNA with oligo $(\mathrm{dT})_{12-18}$ as primers, and the first-strand cDNAs were then amplified by each PCR in the presence of Taq polymerase (Life Technologies) and specific primers. The primers were designed to amplify murine CCL6 referred to the CDNA sequence from the National Center for Biotechnology Information database. The primers were as follows: CCL6 sense, 5'-GACGTCATATGGGCCTCATACAAGAAATG-3' and antisense, 5'-TCGTGGATCCTGCCTGCCCTCCTTCTC-3'; GAPDH sense, 5'-GGTGAAGGTCGGT-GTCAACGGATTT-3' and antisense, 5'-GATGCCAAAGTTGTCATGGATGACC-3'. The PCR reaction was conducted at 35 cycles of $94^{\circ} \mathrm{C}$ for $1 \mathrm{~min}, 55^{\circ} \mathrm{C}$ for $1 \mathrm{~min}$, and $72^{\circ} \mathrm{C}$ for $1 \mathrm{~min}$. Ten microlitres of each PCR product was subjected to electrophoresis on a $2 \%$ agarose gel in the presence of ethidium bromide.

\section{ELISA}

Murine chemokines were quantitated using a standard method of sandwich ELISA as previously described. ${ }^{13,16,21}$ The captured antibodies, detection antibodies and the recombinant cytokines were purchased from R\&D Systems. The ELISAs employed in this study did not cross-react with other murine cytokines, and constantly detected murine cytokines/ chemokines above $10 \mathrm{pg} / \mathrm{ml}$.

\section{Immunohistochemistry}

Cytospin preparations were fixed in 100\% ethanol for $10 \mathrm{~min}$, and the endogenous neutrophil peroxidase was blocked with $0.3 \% \mathrm{H}_{2} \mathrm{O}_{2}$ in methanol. The slides were rehydrated in Tris-buffered saline (TBS) and blocked with 5\% normal goat serum for $20 \mathrm{~min}$ at room temperature. Staining for CCL6 was carried out with anti-CCL6 IgG or control rabbit IgG at $5 \mu \mathrm{g} / \mathrm{ml}$ in TBS-1\% bovine serum albumin for $30 \mathrm{~min}$ at room temperature. After washing with TBS-Tween 20 
$(0.1 \%)$, the slides were incubated with biotinylated goat anti-rabbit IgG antibodies and rinsed. The slides were then incubated for $30 \mathrm{~min}$ with Vectastain Elite ABC Reagent (Vector Laboratories, Burlingame, CA, USA). After washing, the reaction was developed with DAB Substrate Kit (Zymed Laboratories, San Francisco, CA, USA). Counter-staining was done with hematoxylin.

\section{Statistics}

Statistical significance was evaluated by an unpaired Student's $t$-test. $p<0.05$ was regarded as statistically significant. All results are expressed as mean \pm standard error.

\section{Results}

\section{Production of CCL6 during thioglycollate- induced peritonitis}

To determine the production kinetics of CCL6 in this model of acute inflammation, cell-free peritoneal fluids were collected at appropriate time points after thioglycollate injection and CCL6 level in the fluids were measured by ELISA. As shown in Fig. 1, levels of CCL6 reached their peak at $4 \mathrm{~h}$ and the levels were sustained for $48 \mathrm{~h}$ after the injection. Thus, CCL6 increased rapidly in the peritoneum and the level remained elevated even after $48 \mathrm{~h}$ post-injection.

\section{Neutralization of endogenous CCL6 reduces macrophage infiltration}

Experiments were next conducted to determine the role of CCL6 in this model. To address this, endogenous CCL6 was neutralized by administration of anti-CCL6 antiserum prior to thioglycollate injection. The data in Fig. 2 demonstrate that neutralization of CCL6 resulted in significant decreases in the number of infiltrating macrophages at any time examined, ranging from a $34 \%$ reduction to a $48 \%$ reduction ( $43 \%$ reduction on average), as compared with the

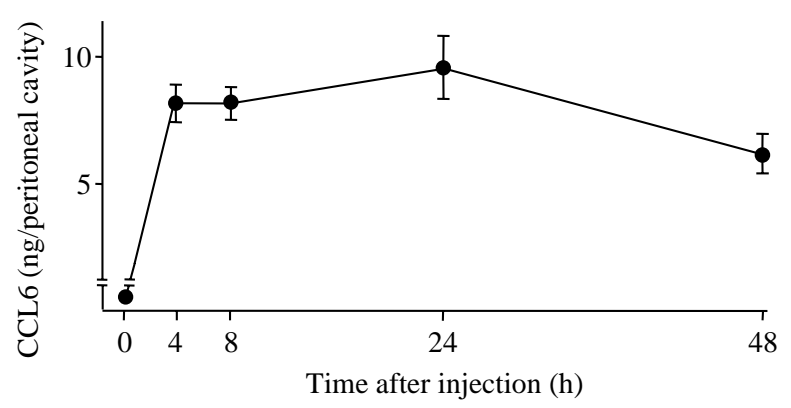

FIG. 1. Production kinetics of CCL6 in the peritoneal fluids after thioglycollate injection. At indicated intervals after thioglycollate injection, mice were euthanized and the peritoneal fluids were harvested (each point, 10-14 mice). CCL6 levels in the peritoneal fluids were measured by ELISA. control. The treatment did not affect the numbers of infiltrating neutrophils, eosinophils, and lymphocytes (Fig. 2). These results indicate that endogenous CCL6 is important in the recruitment of macrophages, but not other cell types.

\section{Effects of anti-CCL6 antiserum on the production of other $\mathrm{CC}$ chemokines}

To determine whether CCL6 could directly or indirectly attract macrophages during inflammation, we examined the peritoneal levels of CCL2, CCL3 and CCL22 known to be involved in the recruitment of macrophages ${ }^{6-10}$ after the treatment with anti-CCL6 antibodies. Consequently, the treatment did not decrease the CCL2 level, but rather augmented CCL2 production, as compared with the control. The CCL22 level was not altered by the treatment (Fig. 3). No appreciable level of CCL3 was detected in the peritoneum (data not shown). Thus, other monocyte chemotactic CC chemokines were not

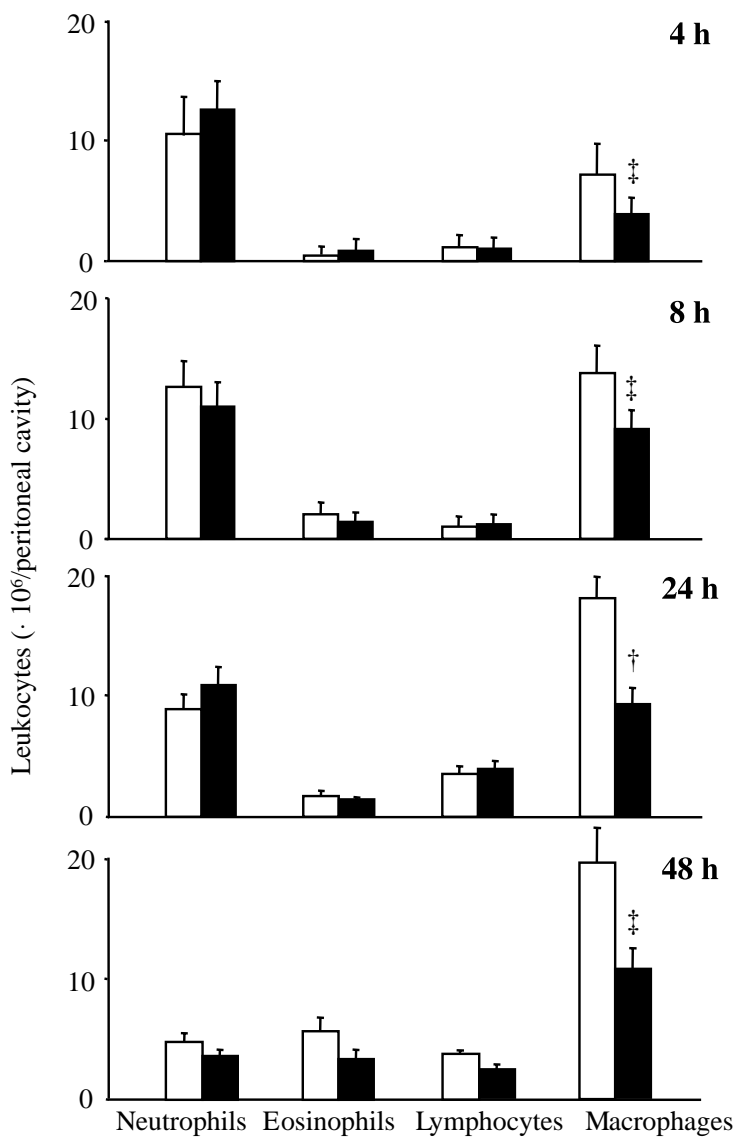

FIG. 2. Effects of neutralization of CCL6 on the leukocyte infiltration induced by thioglycollate. Either $0.5 \mathrm{ml}$ of antiCCL6 antiserum (closed columns) or control serum (open columns) was intraperitoneally injected $2 \mathrm{~h}$ prior to thioglycollate injection. At indicated intervals after thioglycollate injection, mice were euthanized and the infiltrating leukocytes in the peritoneal cavity were harvested (each group, 10 mice). The numbers of neutrophils, eosinophils, lymphocytes and macrophages were then counted. ${ }^{\star} p<0.05$, ${ }^{\dagger} p<0.001$, when compared with control. 


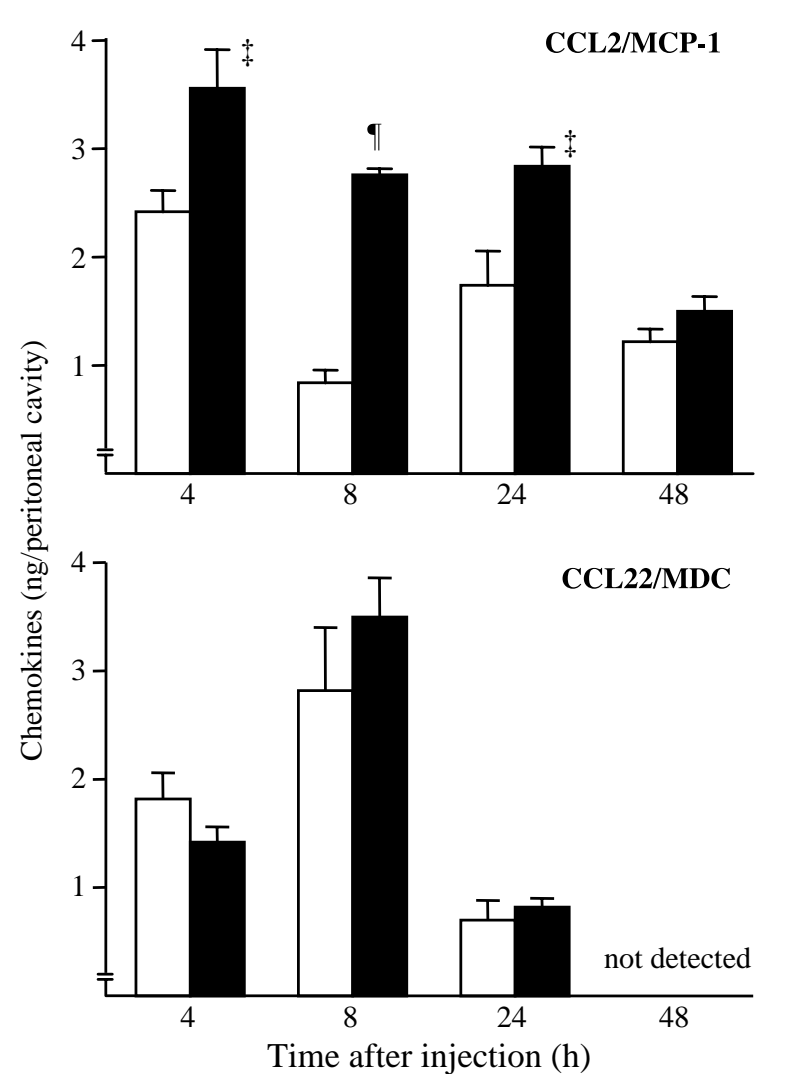

FIG. 3. Effects of anti-CCL6 treatment on CC chemokine production. Either $0.5 \mathrm{ml}$ of anti-CCL6 antiserum (closed columns) or control serum (open columns) was intraperitoneally injected $2 \mathrm{~h}$ prior to thioglycollate injection. At indicated intervals after thioglycollate injection, mice were euthanized and the peritoneal fluids were harvested (each group, 10 mice), and levels of CCL2 and CCL22 were measured by ELISAs. ${ }^{\star} p<0.05,{ }^{\Uparrow} p<0.0001$, when compared with control.

decreased by neutralization of CCL6, indicating that CCL6 may attract monocytes/macrophages independently of other CC chemokines. Anti-CCL6 treatment failed to alter the peritoneal level of CCL1/TCA3, CCL5/RANTES and CCL11/Eotaxin (data not shown).

\section{Cellular source of CCL6}

We next attempted to determine the cellular source of CCL6. First, we isolated peritoneal granulocytes and macrophages following thioglycollate injection, and mRNA expression in the cells was examined by RTPCR. Non-stimulated granulocytes and macrophages were isolated from peripheral blood and the peritoneum from untreated mice, respectively. As shown in Fig. 4, CCL6 mRNA expression was not detected in elicited granulocytes at $4 \mathrm{~h}$ (mean $\%$ eosinophils $=4.4 \%)$ and $8 \mathrm{~h}($ mean $\%$ eosinophils $=13.7 \%$ ) after the injection. No expression was found at $24 \mathrm{~h}$ (mean $\%$ eosinophils $=13.7 \%$; data not shown). In contrast, not only CCL6 mRNA (Fig. 4), but also its protein (Fig. 5) were detected in peripheral granulocytes (mean\% eosinophils $=23 \%$ ), and the protein level in the elicited granulocytes were gradually

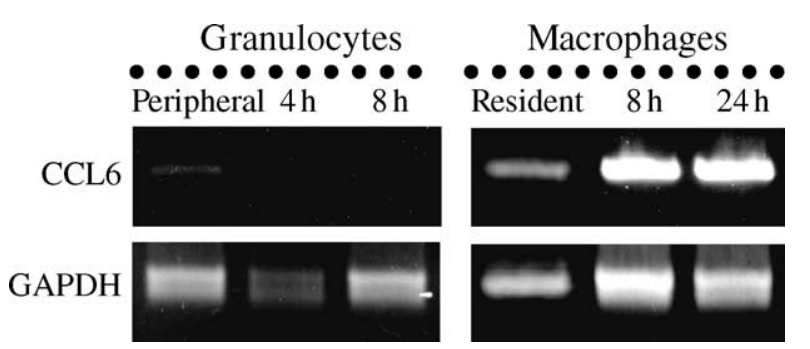

FIG. 4. Expression of CCL6 mRNA in granulocytes and macrophages. Infiltrated granulocytes and macrophages were purified from the elicited leukocytes after thioglycollate injection. Unstimulated granulocytes and macrophages were purified from non-treated peripheral blood and peritoneal cavity, respectively. Total RNA was isolated from the cells and reverse transcribed to cDNA, and the cDNA was amplified by specific primers for murine CCL6 and GAPDH. Shown are representative data from three independent experiments.

decreased as compared with peripheral granulocytes (Fig. 5). On the other hand, both CCL6 mRNA and protein were detected in resident macrophages, and the levels in the elicited macrophages were increased with time during inflammation (Figs 4 and 5). Immunohistochemically, the cells stained with antiCCL6 IgG were eosinophils and macrophages. Neutrophils were not stained with the anti-CCL6 IgG (Fig. 6).

The aforementioned findings suggest a possibility that eosinophils may contribute to the early phase of macrophage influx via releasing the pre-stored CCL6 inside the cells. To address this hypothesis, granulocytes were depleted from mice and peritoneal levels

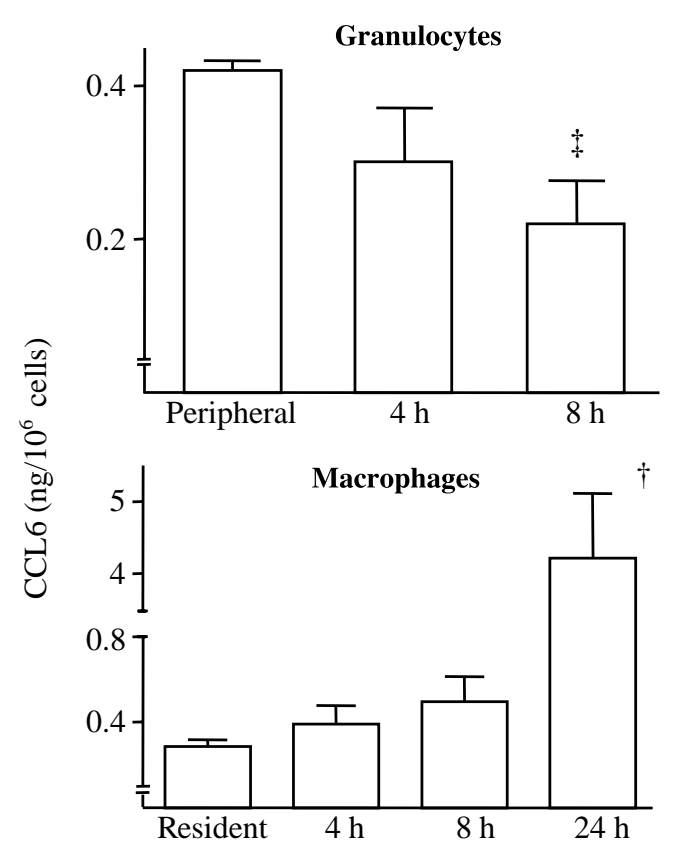

FIG. 5. Intracellular level of CCL6. Granulocytes and macrophages were isolated from peripheral blood or from the peritoneum after thioglycollate injection as indicated. CCL6 levels in cell extracts were measured with ELISA (three to five groups at each time-point). ${ }^{\star} p<0.05,{ }^{\dagger} p<0.001$, when compared with peripheral granulocytes or resident macrophages. 

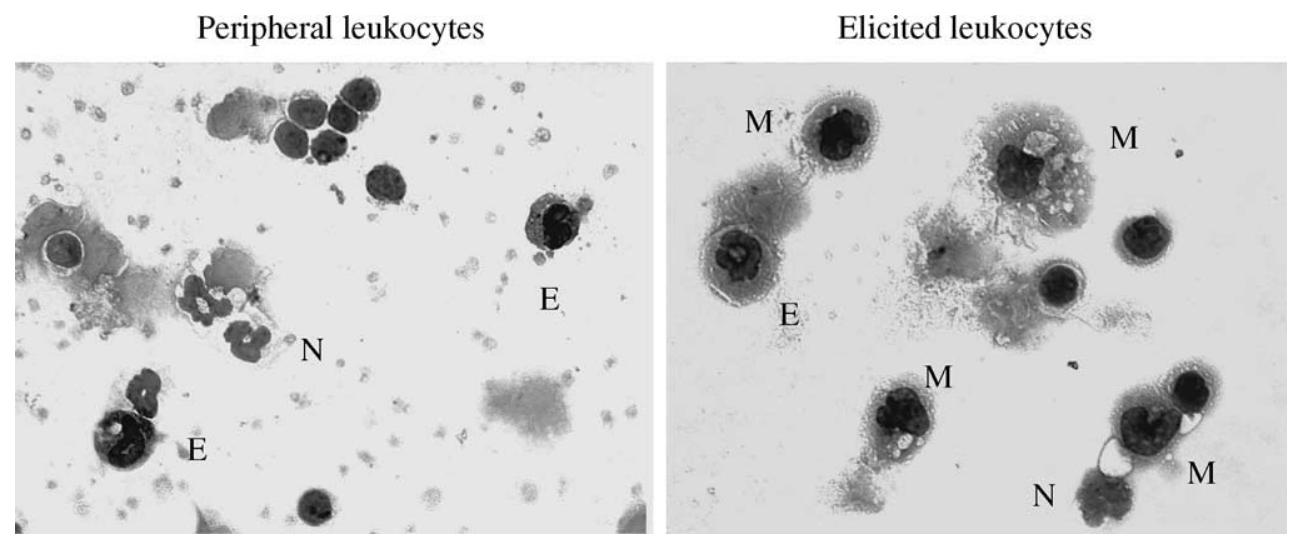

FIG. 6. Cellular source of CCL6. Cytospin preparations of peripheral leukocytes and thioglycollate-elicited leukocytes at $8 \mathrm{~h}$ were stained with anti-CCL6 IgG. E, eosinophils; M, macrophages; N, neutrophils. Shown are representative data from three independent preparations.

of CCL6 in the granulocyte-depleted mice were then measured. Depletion of granulocytes resulted in significant decreases in the number of infiltrating neutrophils and eosinophils at $4 \mathrm{~h}$ and $8 \mathrm{~h}$ after thioglycollate injection (Table 1). Under these conditions, the peritoneal CCL6 levels were measured, which demonstrated that levels of CCL6 were comparable with those in the control (Fig. 7). These findings suggest that CCL6 in eosinophils is not released in the peritoneum in this model.

\section{Discussion}

Inflammation is a series of well-coordinated events that depend on sequential arrival of inflammatory leukocytes to the site of inflammation, in which chemokines play a central role. Our present data suggest that CCL6 is unique in its commitment to the development of inflammation. Production of CCL6 peaked at $4 \mathrm{~h}$ and the level remained elevated even at $48 \mathrm{~h}$ after the injection. Wu et al. demonstrated that high level of CCL6 was detected for up to 10 days in this model. ${ }^{15}$ In contrast, CCL2 and CCL22, known to attract monocytes/macrophages, peaked at $4 \mathrm{~h}$ after the injection and returned to the basal level by $24 \mathrm{~h}$ (own unpublished data). Neutralization of CCL6 resulted in decreases in the number of infiltrating macrophages during the observation periods, but not other cell types, suggesting that CCL6 governs macrophage infiltration from the very beginning to a late period of time, playing a role in the initiation and maintenance of macrophage infiltration.

Macrophage infiltration is induced by multiple CC chemokines, leading to a possibility that CCL6 might induce macrophage infiltration indirectly via the production of other CC chemokines known to be involved in the recruitment of macrophages. A recent investigation has demonstrated that CCL6 neutralization decreased IL-13-induced CCL2 and CCL3 production in the lung. ${ }^{22}$ Neutralization of CCL6 decreased CCL2 production in a murine model of allergic bronchopulmonary aspergillosis. ${ }^{13}$ CCL6 enhanced CCL2 production in a murine model of septic peritonitis. ${ }^{16}$ However, the present data demonstrated that neutralization of CCL6 did not decrease CCL2 as well as CCL22 levels, indicating that CCL6 may attract monocytes/macrophages without reference to other CC chemokines in this particular model. Thus, chemokine-chemokine interaction appears to be different among the types of inflammation.

Interestingly, the CCL2 level was rather increased by neutralization of CCL6. The mechanism by which neutralization of CCL6 increased CCL2 in this model remains to be elucidated. CCL6 is induced by antiinflammatory cytokines interleukin (IL)-4, IL-10 and $\mathrm{IL}-13,{ }^{23}$ raising a possibility that CCL6 may downregulate the production of CCL2. However, CCL6 (1$100 \mathrm{ng} / \mathrm{ml}$ ) failed to decrease the production of CCL2

Table 1. Thioglycollate-induced leukocyte infiltration in granulocyte-depleted mice

\begin{tabular}{lclll}
\hline & Neutrophils & Eosinophils & Lymphocytes & Macrophages \\
\hline $4 \mathrm{~h}$ & & & & \\
RB6-8C5 mAb & $25 \pm 0.15^{\dagger}$ & $0.03 \pm 0.01^{*}$ & $0.72 \pm 0.17$ & $3.67 \pm 0.68$ \\
Control lgG & $4.57 \pm 0.98$ & $0.17 \pm 0.06$ & $0.65 \pm 0.12$ & $3.68 \pm 0.60$ \\
8 h & $0.82 \pm 0.26^{\dagger}$ & $0.14 \pm 0.08^{*}$ & $0.71 \pm 0.16$ & $6.03 \pm 0.87^{*}$ \\
RB6-8C5 mAb & $5.02 \pm 0.96$ & $0.42 \pm 0.10$ & $0.83 \pm 0.14$ & $9.03 \pm 0.95$ \\
Control lgG & &
\end{tabular}

Mice were treated with RB6-8C5 monoclonal antibody (mAb) or control IgG, 1 day prior to thioglycollate injection. At $4 \mathrm{~h}$ and $8 \mathrm{~h}$ after thioglycollate injection, mice were euthanized and the infiltrating leukocytes in the peritoneum were counted (10 mice for each time-point). ${ }^{*} p<0.05,{ }^{\dagger} p<0.001$ versus control. 


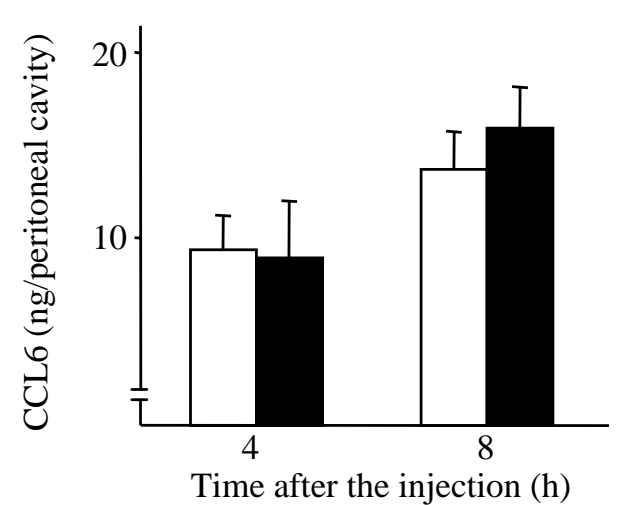

FIG. 7. Peritoneal CCL6 level in granulocyte-depleted mice. Granulocytes were depleted using RB6-8C5 monoclonal antibody. As a control, isotype-matched mouse $\operatorname{lgG}$ was used. At indicated intervals after thioglycollate injection, mice were euthanized and the peritoneal fluids were harvested (each group, 10 mice). CCL6 levels in the peritoneal fluids were measured by ELISA.

when peritoneal macrophages were stimulated with lipopolysaccharide $(1 \mu \mathrm{g} / \mathrm{ml})$ or thioglycollate $(0.4 \%$ $\mathrm{v} / \mathrm{v}$ ) in vitro (data not shown). Thus, a hypothesized immunomodulatory activity of CCL6 is not probable. The reason why the increased level of CCL2 itself did not support the monocyte influx also needs to be determined. Further studies are necessary to clarify these points.

Consistent with a previous report, ${ }^{15}$ cells producing CCL6 were eosinophils and macrophages, as determined by immunohistochemistry. It is known that eosinophils are capable of producing and secreting a number of cytokines and chemokines. ${ }^{24,25}$ In addition to neutrophils, eosinophils begin accumulating in significant numbers in inflammation as shown in the present study and elsewhere. ${ }^{26}$ The present data showed that eosinophils pre-stored CCL6 and the level in granulocytes decreased gradually during inflammation due to the lack of its de novo synthesis. These findings suggest that eosinophils may release CCL6, contributing to the subsequent macrophage infiltration. However, the CCL6 level was not decreased in granulocyte-depleted mice in which the eosinophil influx was significantly decreased, indicating that eosinophils did not release CCL6 in the peritoneum. Therefore, cells other than eosinophils that may include macrophages appear to be the cells responsible for the peritoneal CCL6. It should be noted that granulocyte depletion inhibited the recruitment of macrophages by $33 \%$ at $8 \mathrm{~h}$ despite the number of circulating monocytes being unchanged. This suggests that granulocytes that include neutrophils and eosinophils play a role in macrophage infiltration that is independent of CCL6 production. Neither CCL2 nor CCL3 was the factor, as these chemokine levels were not decreased by granulocyte depletion (data not shown).
In summary, we have demonstrated that CCL6 is an important mediator in the recruitment of macrophages in acute inflammation. Thus, the present data together with those in chronic inflammation models suggest that CCL6 may have function in both acute and chronic inflammatory responses. Acute inflammation is tightly linked to T-cell-mediated chronic inflammation, suggesting a broad activity of CCL6 in the development and maintenance of inflammatory disorders.

ACKNOWLEDGEMENTS. This work was supported in part by grants from The Ministry of Education, Science, Sports and Culture, and The Ministry of Health and Welfare, Japan.

\section{References}

1. Luster AD. Chemokines - chemotactic cytokines that mediate inflammation. N Engl J Med 1998; 338: 436-445.

2. Feng L. Role of chemokines in inflammation and immunoregulation. Immunol Res 2000; 21: 203-210.

3. Baggiolini M. Chemokines and leukocyte traffic. Nature 1998; 392: 565-568.

4. Rot A, Von Andrian UH. Chemokines in innate and adaptive host defense: basic chemokinese grammar for immune cells. Annu Rev Immunol 2004; 22: 891-928.

5. Gerard C, Rollins BJ. Chemokines and disease. Nat Immunol 2001; 2: $108-115$.

6. Matsukawa A, Miyazaki S, Maeda T, et al. Production and regulation of monocyte chemoattractant protein-1 in lipopolysaccharide- or monosodium urate crystal-induced arthritis in rabbits: roles of tumor necrosis factor alpha, interleukin-1, and interleukin-8. Lab Invest 1998; 78: $973-$ 985.

7. Matsukawa A, Hogaboam CM, Lukacs NW, Lincoln PM, Strieter RM, Kunkel SL. Endogenous monocyte chemoattractant protein-1 (MCP-1) protects mice in a model of acute septic peritonitis: cross-talk between MCP-1 and leukotriene B4. J Immunol 1999; 163: 6148-6154.

8. Matsukawa A, Hogaboam CM, Lukacs NW, Lincoln PM, Evanoff HL, Kunkel SL. Pivotal role of the CC chemokine, macrophage-derived chemokine, in the innate immune response. J Immunol 2000; 164: $5362-5368$

9. Ajuebor MN, Flower RJ, Hannon R, Christie M, Bowers K, Verity A, Perretti M. Endogenous monocyte chemoattractant protein-1 recruits monocytes in the zymosan peritonitis model. J Leukoc Biol 1998; 63 : $108-116$.

10. Ajuebor MN, Das AM, Virag L, Szabo C, Perretti M. Regulation of macrophage inflammatory protein- 1 alpha expression and function by endogenous interleukin-10 in a model of acute inflammation. Biochem Biophys Res Commun 1999; 255: 279-282.

11. Orlofsky A, Berger MS, Prystowsky MB. Novel expression pattern of a new member of the MIP-1 family of cytokine-like genes. Cell Regulat 1991; 2: 403-412.

12. Belperio JA, Dy M, Burdick MD, Xue YY, Li K, Elias JA, Keane MP. Interaction of IL-13 and C10 in the pathogenesis of bleomycin-induced pulmonary fibrosis. Am J Respir Cell Mol Biol 2002; 27: 419-427.

13. Hogaboam CM, Gallinat CS, Taub DD, Strieter RM, Kunkel SL, Lukacs NW. Immunomodulatory role of $\mathrm{C} 10$ chemokine in a murine model of allergic bronchopulmonary aspergillosis. J Immunol 1999; 162: 60716079

14. Asensio VC, Lassmann S, Pagenstecher A, Steffensen SC, Henriksen SJ, Campbell IL. C10 is a novel chemokine expressed in experimental inflammatory demyelinating disorders that promotes recruitment of macrophages to the central nervous system. Am I Pathol 1999; 154: $1181-1191$

15. Wu Y, Prystowsky MB, Orlofsky A. Sustained high-level production of murine chemokine C10 during chronic inflammation. Cytokine 1999; 11: $523-530$.

16. Steinhauser ML, Hogaboam CM, Matsukawa A, Lukacs NW, Strieter RM, Kunkel SL. Chemokine C10 promotes disease resolution and survival in an experimental model of bacterial sepsis. Infect Immun 2000; 68: 6108-6114.

17. Hestdal K, Ruscetti FW, Ihle JN, et al. Characterization and regulation of RB6-8C5 antigen expression on murine bone marrow cells. J Immunol 1991; 147: 22-28

18. Hamaguchi-Tsuru E, Nobumoto A, Hirose N, et al. Development and functional analysis of eosinophils from murine embryonic stem cells. $\mathrm{Br}$ J Haematol 2004; 124: 819-827. 
19. Tateda K, Moore TA, Deng JC, et al. Early recruitment of neutrophils determines subsequent T1/T2 host responses in a murine model of Legionella pneumophila pneumonia. J Immunol 2001; 166: 3355-3361.

20. Singer M, Lefort J, Vargaftig BB. Granulocyte depletion and dexamethasone differentially modulate airways hyperreactivity, inflammation, mucus accumulation, and secretion induced by rmIL-13 or antigen. Am J Respir Cell Mol Biol 2002; 26: 74-84.

21. Evanoff HL, Burdick MD, Moore SA, Kunkel SL, Strieter RM. A sensitive ELISA for the detection of human monocyte chemoattractant protein-1 (MCP-1). Immunol Invest 1992; 21: 39-45.

22. Ma B, Zhu Z, Homer RJ, Gerard C, Strieter R, Elias JA. The C10/CCL6 chemokine and CCR1 play critical roles in the pathogenesis of IL-13induced inflammation and remodeling. I Immunol 2004; 172: $1872-$ 1881
23. Orlofsky A, Wu Y, Prystowsky MB. Divergent regulation of the murine CC chemokine C10 by $\mathrm{Th}(1)$ and $\mathrm{Th}(2)$ cytokines. Cytokine 2000; 12: $220-228$.

24. Lacy P, Moqbel R. Eosinophil cytokines. Chem Immunol 2000; 76: 134155

25. Oliveira SH, Taub DD, Nagel J, et al. Stem cell factor induces eosinophil activation and degranulation: mediator release and gene array analysis. Blood 2002; 100: 4291-4297.

26. Melnicoff MJ, Horan PK, Morahan PS. Kinetics of changes in peritoneal cell populations following acute inflammation. Cell Immunol 1989; 118 : $178-191$.

\section{Received 18 August 2004}

Accepted 10 September 2004 


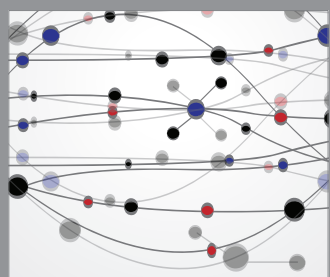

The Scientific World Journal
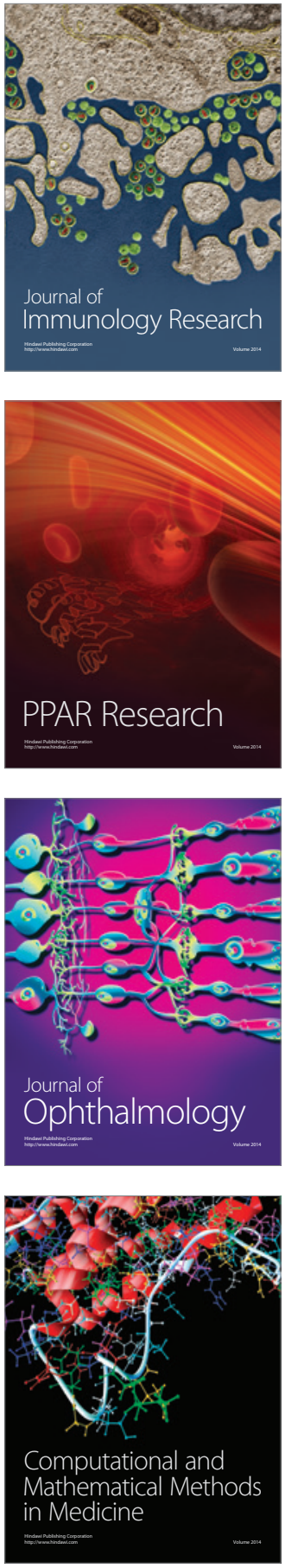

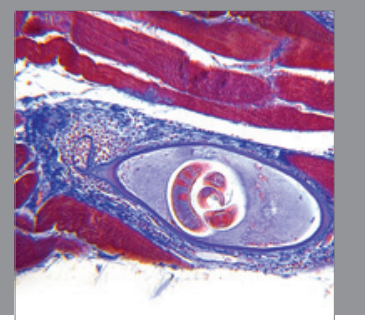

Gastroenterology

Research and Practice
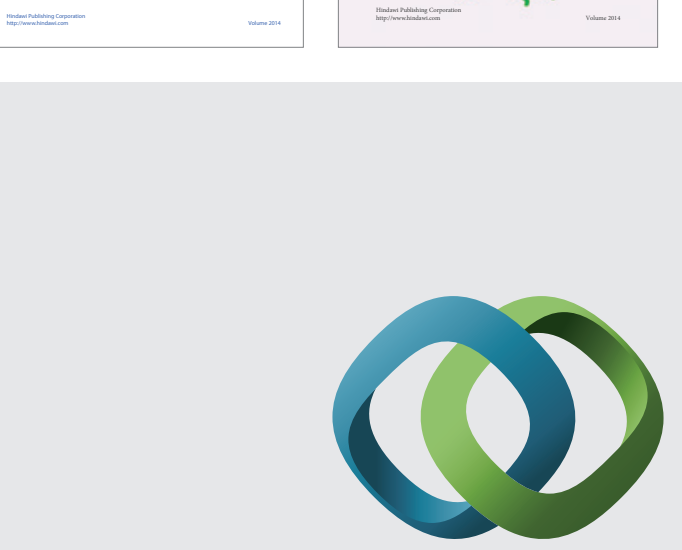

\section{Hindawi}

Submit your manuscripts at

http://www.hindawi.com
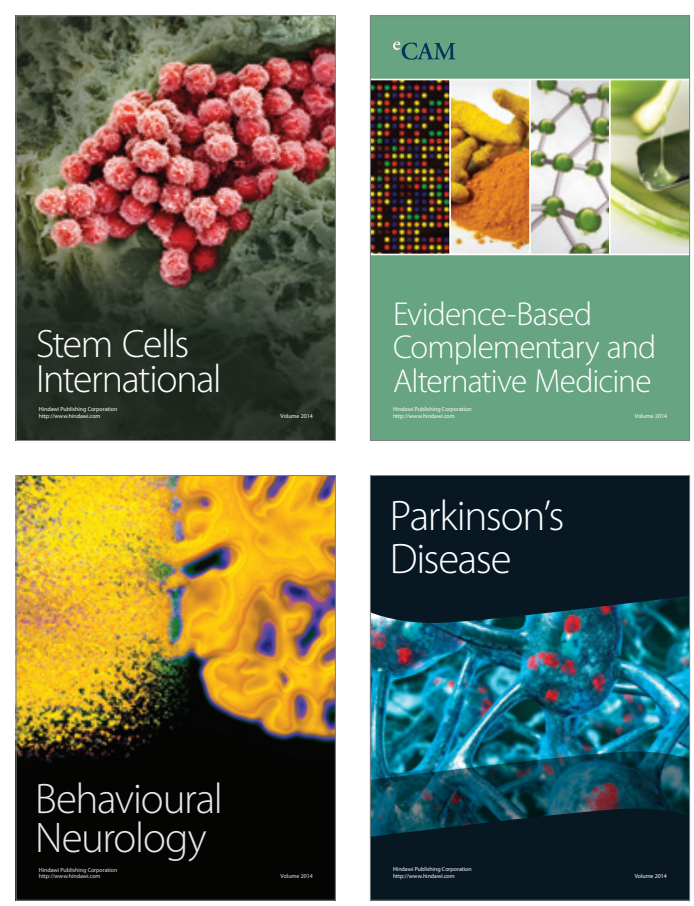

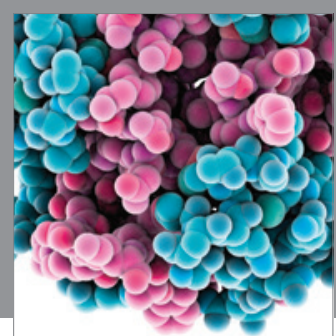

Journal of
Diabetes Research

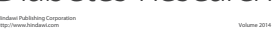

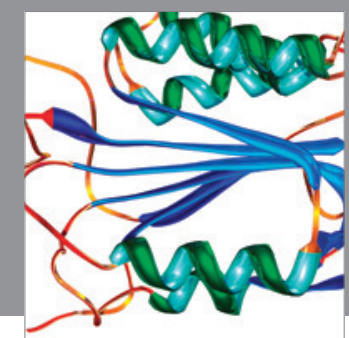

Disease Markers
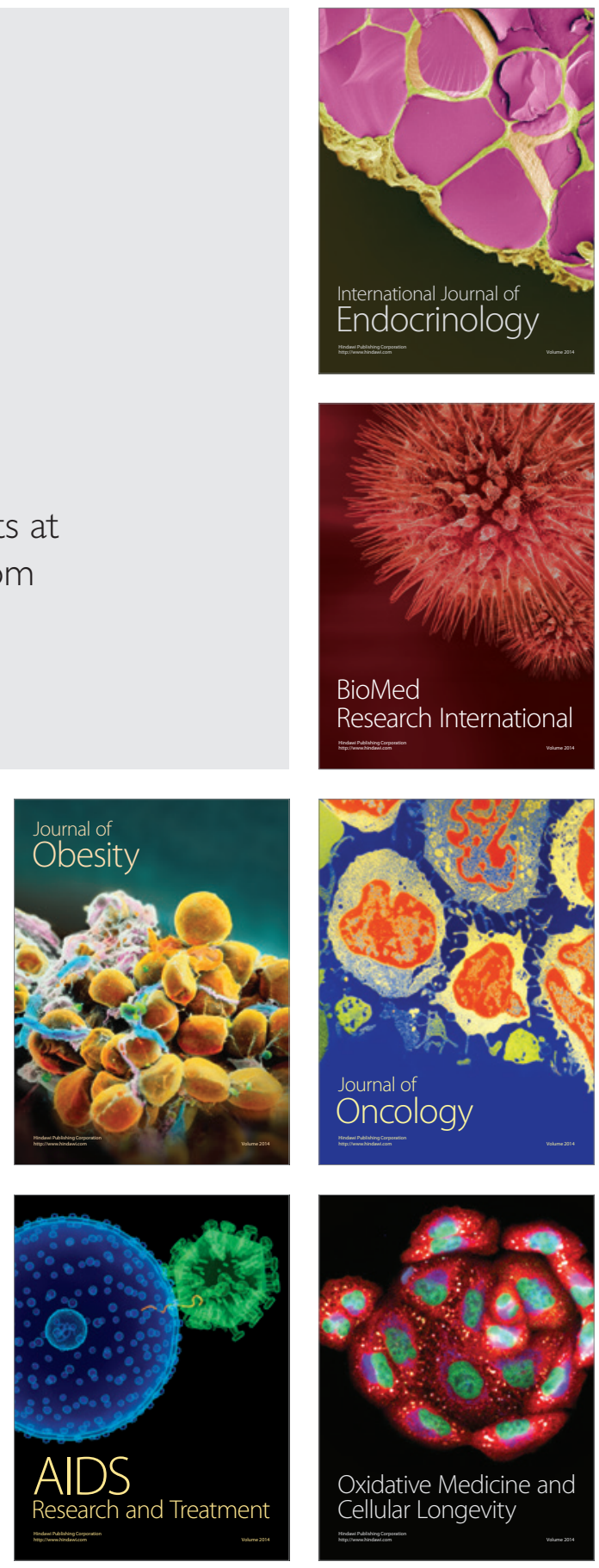